\title{
LA SOCIEDAD SECRETA Y LA REBELIÓN DE LOS MAGOS: UNA APROXIMACIÓN A LOS SIETE LOCOS Y LOS LANZALLAMAS*
}

\author{
. . l'imaginaire, bien loin \\ d'être vaine passion, est action \\ euphémique et transforme le monde \\ selon l'Homme de Désir. \\ GILBERT DURAND, Les structures \\ anthropologiques de l'imaginaire.
}

Novelas de múltiples planos, densas, complejas, Los siete locos (1929) y su continuación Los lanzallamas (1931) ${ }^{1}$ son sin duda las más significativas y originales de Roberto Arlt, las que ofrecen también mayores retos a la crítica. Si en su primera novela, El juguete rabioso, publicada en 1926 - relato emparentado con la novela de aprendizaje-, aparecen ya en germen varias de las constantes temáticas y de estilo de su narrativa posterior, su construcción sobria y nítida, su desarrollo lineal y verosímil (aspectos sin duda vinculados con la percepción de un mundo todavía estable y equilibrado), contrastan notablemente con el universo caótico de Los siete locos y Los lanzallamas. Éstas nos introducen en efecto en un mundo heterogéneo, ambiguo, que oscila entre el folletín y la aventura, entre las reiteradas incursiones en las zonas subconscientes de los personajes y la fantasía política con el extraño proyecto de revolución que persigue la Sociedad Secreta organizada por el no menos extraño Astrólogo². La trayectoria interior del protagonis-

* El artículo que aquí ofrezco es parte de un estudio mayor, en vías de publicación, sobre la obra del escritor argentino Roberto Arlt (1900-1942).

${ }^{1}$ Seguimos aquí la edición prologada por Adolfo Prieto en Biblioteca Ayacucho, Caracas, 1978, 456 pp. Utilizaremos para Los siete locos y Los lanzallamas las siguientes abreviaturas: $S l$ y $L l$.

${ }^{2}$ Tal vez no sea tan casual el que Arlt haya escogido como cabecilla del 
ta, Remo Erdosain - "inventor fracasado" y "delincuente al margen de la cárcel", se dice en las primeras páginas de Los siete locos-, corre parejo y se entreteje con la historia de los demás "locos" que integran la Sociedad Secreta. Aparece una gran diversidad de personajes secundarios, algunos fugazmente como el Buscador de Oro o El Mayor, otros mejor delineados (pensamos, en particular, en Haffner, apodado el "Rufián Melancólico", antiguo profesor de matemáticas dedicado ahora al negocio de la prostitución, en Hipólita o la Coja -ex-sirvienta y ex-prostituta-, en el místico Ergueta), personajes que siguen en buena medida destinos paralelos que sólo convergen de manera circunstancial en el asunto de la Sociedad Secreta. Un poco a la manera de Gide que, precisamente por aquellos años, sostenía en Los monederos falsos (1925) que "todo debía entrar en una novela"'3, Arlt abandona la conocida prescripción naturalista de "la tranche de vie" en un sentido temporal y presenta ahora, por el contrario, una visión en profundidad. En lugar de reconstruir "vidas", las novelas penetran reiteradamente en los "estados de conciencia" ( $S l$, p. 4) de los personajes, en la "zona de la angustia"' ( $S l$, p. 5), y dan asimismo cabida a sus sueños y fantasías, sus alucinaciones $u$ obsesiones. Los personajes de Los siete locos y Los lanzallamas parecen hallarse al final de una atormentada y errática trayectoria cuyas etapas anteriores ignoramos o de las cuales sólo se nos entregan algunos fragmentos.

grupo a un astrólogo. En efecto, en uno de sus primeros textos, "Las ciencias ocultas en la ciudad de Buenos Aires" - publicado en 1920 y afortunadamente reeditado en Roberto ARLt, Obra completa, prólogo de Julio Cortázar, Buenos Aires, 1981, 2 ts.-, Arlt muestra su temprana afición por estas disciplinas exponiendo con evidente interés, como lo testimonian las numerosas lecturas que ha hecho sobre el tema, algunas de sus bases. Sin embargo, de manera paralela, denuncia el "pseudo espiritualismo" que prevalece en los centros espiritistas, la "sabiduría de los astrólogos logreros", y ataca en particular la Doctrina Secreta, obra capital de la autora y fundadora de la Sociedad Teosófica en 1875, Elena Petrona Blavatsky. Aunque hay que cuidarse de establecer correspondencias demasiado estrechas entre este ensayo y las novelas que nos ocupan, parece indudable que las experiencias de Arlt en estos centros sirvieron de punto de partida para la creación de la Sociedad Secreta y del Astrólogo.

3 'Le grand défaut de cette école [o sea la naturalista, dice un personaje de la novela], c'est de couper sa tranche toujours dans le même sens; dans le sens du temps, en longueur. Pourquoi pas en largeur? ou en profondeur? Pour moi, je voudrais ne pas couper du tout. Comprenez-moi: je voudrais tout y faire entrer dans ce roman' (Les faux-monnayeurs, Gallimard, Paris, 1983, p. 184). 
Tomada al pie de la letra, parece imposible unificar en una visión coherente y verosímil la propuesta revolucionaria del Astrólogo. Como lo advierte Adolfo Prieto, el asunto de la Sociedad Secreta y todo lo que tiene que ver con él configura en las novelas una "poderosa metáfora", un "orden de flancos abiertos" que admite distintas lecturas". Las críticas, válidas y en buena medida proféticas, que formulan los personajes en torno a la naciente sociedad capitalista porteña, se combinan con los detalles de la organización revolucionaria y con lás propuestas fantasiosas, mágicas, que pretenden acabar con dicha sociedad. Sorprende, por lo mismo, el empeño de la crítica arltiana por descontextualizar las “ideas"' políticas de los personajes, ordenarlas, sistematizarlas, convertirlas incluso en un testimonio directo y unívoco del autor, olvidando sin embargo algo esencial: la estrecha vinculación entre el proyecto revolucionario y los "locos" de la novela (los marginados o "las fuerzas perdidas", $S l$, p. 98, de la sociedad a las que busca seducir por todos los medios el Astrólogo) a quienes va dirigido ${ }^{5}$. Las numerosas referencias a la historia contemporánea - subrayadas en particular por los comentarios y notas a pie de página de un personaje que se autodenomina "cronista" y "comentador" - , los posibles modelos "reales" de revolución

4 Prieto, "Prólogo" a R. ARlt, Los siete locos y Los lanzallamas, p. xxiv.

${ }^{5}$ El primer libro que se publica sobre Arlt, la conocida biografía de RAúl LARRA, Roberto Arlt, el torturado (Futuro, Buenos Aires, 1950), aunque tiene el mérito de llamar la atención sobre el autor, incurre en asimilaciones de diversos órdenes entre los personajes, sus características psicológicas, sus ideas (principalmente sus ideas políticas; ver el capítulo intitulado "El escritor y la política"') y el autor. Estudios más recientes inciden sin embargo en un error semejante: discutir las "ideas", tratar de encontrar una coherencia a las mismas con criterios de verosimilitud. Es el caso por ejemplo de BEATRIz PASTOR en Roberto Arlty la rebelión alienada, Hispamérica, Gaithersburg, 1980. (Véase en particular el capítulo dedicado a "La alternativa revolucionaria", donde Pastor hace una crítica ideológica del programa revolucionario del Astrólogo). También José Amícola, en su documentado estudio, Astrología y fascismo en la obra de Roberto Arlt (Weimar, Buenos Aires, 1984), relaciona estrechamente las distintas corrientes políticas, ideológicas y filosóficas que recorren los años veinte con los planteamientos de los "locos" en el seno de la Sociedad Secreta. Estas correspondencias, que iluminan sin duda el contexto en el que Arlt escribe sus novelas, no son sin embargo suficientes para explicar el proyecto de los "locos" tal y como aparece en la ficción. Como dice con mucha razón FERNANDO AlEGRf́, "Arlt intriga a los críticos y estudiosos de su obra. Ellos quisieran reducir sus novelas a un sistema de premisas estéticas y éticas, presentar sus novelas como piezas de una estructura ideológica. Pero Arlt se resbala y escapa' (Nueva historia de la novela hispanoamericana, Ediciones del Norte, Hanover, 1986, p. 175). 
y de organizaciones secretas no interesan en tanto documento o verdad objetiva. Al incorporarse a la ficción, la realidad histórica se supedita, como intentaremos mostrarlo, a una verdad imaginativa. El presente análisis procura situar el debate central de Los siete locos y Los lanzallamas en la ficción: ¿qué pretende en realidad el Astrólogo, jefe e ideólogo indiscutible del grupo? ¿cuál es la eficacia propia de su plan revolucionario y qué función cumple en la trama narrativa?

La Sociedad Secreta persigue un doble objetivo: por una parte, la desaparición o, mejor dicho, la destrucción ciega, apocalíptica de la sociedad de explotación capitalista y, por otra, el restablecimiento de la fe perdida, de un "dios creador del cielo y de la tierra" ( $L l$, p. 202), condición necesaria para sanar el alma enferma del hombre contemporáneo. En el tono vehemente que suele caracterizarlo, el Astrólogo afirma: "Yo creo en un único deber: luchar para destruir esta sociedad implacable. El régimen capitalista en combinación con los ateos han convertido al hombre en un monstruo escéptico, verdugo de sus semejantes por el placer de un cigarro, de una comida o de un vaso de vino" ( $L l$, p. 203). El "'mal del siglo" que representa la "irreligión" ( $S l$, p. 59) o, para decirlo con una fórmula célebre de Nietzche, la "muerte de Dios", el abismo cada vez mayor entre ciencia y fe, se asocian con otras miserias de la modernidad: los adelantos tecnológicos que esclavizan al hombre y lo deshumanizan, el crecimiento anárquico de las urbes que denuncia en particular el Buscador de Oro, otro de los integrantes del grupo. La ausencia de valores o de ideales que prevalece en la evocación que hace el Astrólogo del primer tercio del siglo $\mathrm{xx}$ parece ser el factor decisivo del desamparo, de la angustia de los hombres, o de lo que llama también "la enfermedad metafísica de todo hombre”' ( $S l$, p. 93). La Sociedad Secreta y el programa formulado por el Astrólogo intentan ser, entonces, una solución o una respuesta a estos males. Respuesta sin embargo contradictoria, dispar, difícilmente viable, en la que todo cabe ${ }^{6}$ l las fantasías omnipotentes, los sueños " extraordina-

${ }^{6}$ Es muy probable que Arlt haya descubierto, como se desprende de su ensayo sobre las ciencias ocultas, en las logias teosóficas que conoció y desde luego en La Doctrina Secreta de la Sra. Blavatsky, esta búsqueda deliberada de la confusión que parece perseguir el Astrólogo. En el inciso dedicado a los "fraudes y la Doctrina Secreta", después de denunciar "las aparentes maravillas", "los pretendidos milagros", las numerosas contradicciones de la obra, Arlt concluye: "[...] se podría decir al que quisiera descubrir la verdad en este caos: No caves más porque encontrarás la locura"' (Obra Completa, t. 2, p. 23). 
rios", la "mentira metafísica", y una desconcertante y posiblemente irónica amalgama entre distintas ideologías: "No sé [argumenta el Astrólogo] si nuestra sociedad será bolchevique o fascista. A veces me inclino a creer que lo mejor que se puede hacer es preparar una ensalada rusa que ni Dios la entienda"' $(S l$, p. 22). Poco después, afirma también: "Seremos bolcheviques, católicos, fascistas, ateos, militaristas, en diversos grados de iniciación" ( $S l$, p. 97) $)^{7}$. En un mismo terreno se codean Lenin, Mussolini, Henry Ford, Al Capone y distintos modelos de sociedad secreta, contemporáneos como el Ku-Klux-Klan o pasados como la sociedad organizada en el siglo Ix por un bandido árabe, Abdala-Aben-Maimun. En todos ellos encuentra el Astrólogo algún aspecto digno de ser tomado en cuenta, independientemente de los fines perseguidos por cada uno de los aludidos. Si la voluntad, la fuerza que abriga la empresa de Lenin lo convierten en "el último dios terrestre que pasó por el mundo" ( $\mathrm{Ll}$, p. 237), de Mussolini, que ha sabido "apoderarse del alma de una generación", admira el autoritarismo, la "eficacia del bastón en la espalda de los pueblos"' ( $S l$, p. 95). Una de las ideas medulares de la teoría del Astrólogo es la mentira o, mejor, el "poder de la mentira"' ( $S l$, p. 108), idea que pone en práctica en el seno mismo de la Sociedad Secreta. En efecto, los engaños, las comedias, las "farsas" - así se titula justamente una de las primeras reuniones del grupo en la quinta del Astrólogo en Temperley, en las afueras de Buenos Aires- están a la orden del día. Semejante al titiritero que imagina ser en una larga meditación nocturna ( $S l$, pp. 164-165), el Astrólogo mueve los hilos de la historia, pero permanece en la sombra, guardando siempre, como dice uno de los personajes, "el secreto de sus procedimientos"' ( $S l$, p. 104). No puede pasarse por alto la constante manipulación de la información que el Astrólogo entrega a cada uno de los aspirantes a revolucionario, la estrecha vigilancia que ejerce sobre algunos de ellos $\mathrm{y}$, finalmente, la sospecha general a que dan lugar tales métodos.

${ }^{7}$ En sus respectivos estudios, Amícola y Pastor asocian este eclecticismo ideológico con los contradictorios programas del fascismo temprano, o sea entre 1920 y 1930. JosÉ BIANCO, por su parte, alude al "carácter escénico de la dictadura [de Perón], a sus ficciones que no podían creerse y eran creídas", y agrega: "A mi juicio, nada se parece tanto a las ficciones del peronismo como las ficciones de R. Arlt" ("En torno a Roberto Arlt", CasA, 1961, núm. 5, pp. 50-51). No puede en efecto olvidarse que, en buena medida, las fórmulas políticas híbridas anunciadas por el Astrólogo tendrán éxito en la Argentina de Perón algunos años después. 
Haffner, Erdosain o el Abogado no saben a ciencia cierta dónde ubicar al Astrólogo, qué propósitos se esconden tras su singular y enigmático "rostro romboidal"' ( $S l$, p. 20). Al principio de Los siete locos, Erdosain, que lo conoció en una Sociedad Teosófica, conjetura que se trata de un "delegado bolchevique"' ( $S l$, p. 19). Poco después, en una conversación a solas con Haffner, al término de la primera entrevista en la casa del Astrólogo, Erdosain le pregunta a quemarropa:

-Pero usted, en su interior, ¿qué piensa del Astrólogo?

-Que es un maniático que puede tener o no éxito.

- Pero sus ideas. . .

-Algunas son embrolladas, otras claras, y francamente, yo no sé hasta dónde quiere apuntar ese hombre. Unas veces usted cree estar oyendo a un reaccionario, otras a un rojo, y, a decir la verdad, me parece que ni él mismo sabe lo que quiere $(S l$, p. 31$)$.

El Abogado, partidario pasajero de la Sociedad Secreta, se irrita ante las incongruencias de su pensamiento y rompe de manera abrupta las reglas del juego que todos observan; primero, al discrepar abiertamente con sus ideas y, después, al abofetearlo no sin antes tildarlo de "comediante", "cínico" y " aventurero" ( $\mathrm{Ll}$, p. 242). El mismo desconcierto se apodera del lector al no lograr conciliar los distintos papeles asumidos por el Astrólogo. La dimensión política o "revolucionaria" de la figura del Astrólogo parece haber opacado otros de sus atributos que refuerzan, por una parte, el misterio que envuelve su persona y que, por otra, lo acercan al sustrato fantasioso de su proyecto. Al dudoso "oficio" de astrólogo, hay que agregar su manifiesta afición por las ciencias ocultas, perceptible, por ejemplo, en su admiración por el místico Swedenborg o en el hecho de considerarse literalmente un "iluminado" ( $S l$, p. 162) ${ }^{8}$. Su estrafalaria vestimenta - ¿otro disfraz? - detalle aparentemente secundario, desorienta, se convierte en un elemento más de misterio e impide precisar su origen: “ " . . . ] apareció por la puerta la gigantesca figura del Astrólogo, cubierto con un guardapolvo amarillo y la galera echada sobre la frente, sombreándole el anchuroso rostro romboidal' ' $(S l$, p. 20). Poco

${ }^{8}$ Los demás "locos" comparten en mayor o menor medida su misticismo; así lo afirma el Astrólogo a Erdosain: "Nosotros somos místicos sin saberlo. Místico es el Rufián Melancólico, místico es Ergueta, usted, yo, ella [Hipólita] y ellos [...] Necesitamos de una religión para salvarnos de esa catástrofe que ha caído sobre nuestra cabeza"' (Sl, p. 59). 
después, el narrador apunta que el guardapolvo amarillo del Astrólogo "parecía la vestimenta de un sacerdote de Buda" ( $S$, p. 21), y en las últimas páginas de Los lanzallamas se recurre de nuevo a otro símil: "[. . . ] visto de espaldas, con su sombrero plano, parecía un profesor del rito protestante" ( $L l$, p. 381). El Astrólogo se parece, en efecto, a varios posibles modelos que, apenas esbozados, se esfuman. Pero permanece la duda en torno a su identidad, de perfiles borrosos, y a sus propósitos últimos. Nada en la novela autoriza en efecto a formular un juicio definitivo en torno a la figura ambigua del Astrólogo. Éste expone sus ideas a lo largo de interminables reuniones en Temperley sin que se interponga alguna mirada privilegiada, omnisciente, que se aparte de la perspectiva del personaje, enjuiciándolo o enfocándolo desde un punto de vista distinto. De ahí, en buena medida, que el carácter impredecible, versátil de la personalidad del Astrólogo se sostenga hasta el término de Los lanzallamas, y que la historia de la Sociedad Secreta, ajena a las jerarquizaciones, permanezca asimismo abierta a las interpretaciones. Si volvemos ahora a nuestro punto de partida, la mentira esencial sobre la que descansa la teoría del Astrólogo es la mentira "elocuente, enorme, trascendental", "extraordinaria", cercana al prodigio, a la que llama "'mentira metafísica"' ( $S l$, p. 93), capaz de devolverles a los hombres la felicidad perdida. Ésta responde, pues, a la enfermedad diagnosticada por el Astrólogo y se origina en su creencia de que "sólo los prodigios conseguirán emocionar" (Sl, p. 78) al hombre. Con el propósito de sacudir la inercia y el aburrimiento de sus prosélitos y de comprobar así su teoría, el Astrólogo (del que se dice también que es un "maestro en sorpresas", $S l$, p. 103), invita a un mayor apócrifo ${ }^{9}$ a la primera reunión del grupo para representar el papel del militar encargado de infiltrar la Sociedad Secreta en el ejército y deja que el Buscador de Oro los maraville con su historia del oro coloidal. Al enterarse poco después de que tal historia es una invención más para cautivarlos, Erdosain se queja amargamente: "[creía] que entre tantas mentiras, ésa sería una de las pocas verdades"' ( $S l$, p. 113). A pesar de que el clima de desconfianza anteriormente apuntado pone en entredicho la complicidad real de los integrantes de la Sociedad Secreta, no pue-

9 Sumándose, al parecer, a la "farsa" montada por el Astrólogo, el cronista interviene en una nota a pie de página para ¿aclarar? que el "Mayor no era apócrifo, sino auténtico, y que mintió al decir que representaba una comedia" (Sl, p. 108). 
de ignorarse que los discursos del Astrólogo dedicados a la destrucción de la actual sociedad y al plan quimérico de otra futura, discursos que pertenecen de lleno a un mundo en que la fantasía tiene libre curso, reciben la entusiasta adhesión de los miembros del grupo. Alternan las fábricas de gases capaces de exterminar a una población entera en unos cuantos minutos, las guillotinas, el "Rayo de la muerte"' que hace "saltar en cascajos las ciudades de portland", que esteriliza campos y "convierte en cenizas las razas y los bosques"' ( $S l$, pp. 179, 180), las bíblicas "lluvias de fuego" ( $L l$, p. 335), con la falsa resurrección de lo sagrado mediante milagros apócrifos, dioses inventados, "mentiras perfectas" destinadas a las "enfermedades más fantásticas del entendimiento y del alma' ( $S l$, p. 181); un mundo, pues, poblado de deseos y sueños omnipotentes en donde la magia - magia circunscrita a las palabras - recobra sus antiguos poderes ${ }^{10}$. Erdosain, por ejemplo, se imagina convertido en "Dueño del Universo": "Héroes de todas las épocas sobrevivían en él. Ulises, Demetrio, Aníbal, Loyola, Napoleón, Lenin, Mussolini, cruzaban ante sus ojos como grandes ruedas ardientes, y se perdían en un declive de la tierra solitaria bajo un crepúsculo que ya no era terrestre",

${ }^{10}$ Es evidente la cercanía existente entre las fantasías destructivas, las persecuciones mágicas (rayos, electricidad, sistemas), y en definitiva el poder ilimitado pero ficticio que creen poseer los "locos" de la novela, con las fantasías esquizofrénicas. Al hablar del "yo en la condición esquizoide", RonAlD D. LAING puntualiza: "Sólo es omnipotente y libre en la fantasía [...]. La ilusión de la omnipotencia y de la libertad puede mantenerse solamente dentro del círculo mágico de su propio 'cierre' en la fantasía" (El yo dividido, F.C.E., México, 1974, pp. 79-80). Por otra parte, resulta interesante acotar que las fantasías de los "locos", sustitutivas como se verá de la revolución "real", coinciden con la "magia esquizofrénica" estudiada por GÉzA RóHEIM en $L a$ magia y la esquizofrenia, Paidós, Buenos Aires, 1959. Róheim advierte, en primer término, que "las formas básicas u originales de la fantasía mágica y esquizofrénica nacen de las mismas raíces"' (p. 13). Sin embargo, frente a las "fantasías mágicas" del hombre primitivo, fase inicial de cualquier actividad, aquélla se singulariza por ser únicamente "magia imaginada" a la que "no sucede una acción en la realidad" (id.), debido a la desintegración de la identidad del esquizofrénico. Los personajes carentes de identidad que deambulan por la novela parecen movilizar lo que llama Róheim "el principio de la magia", principio que trata "el mundo externo como si éste fuera regido por nuestros deseos o impulsos o emociones" (p. 79). Leo NAvratil en Esquizofrenia $y$ arte (Seix Barral, Barcelona, 1972) plantea también que cuando se desencadenan miedos irracionales -el "terror" es precisamente la imagen que mejor define el espacio de estas novelas- "sólo pueden mantenerse a raya por medio de signos y acciones mágicas” (p. 66). 
(Sl, p. 179). Por su parte el Astrólogo, en el colmo del delirio, profiere la siguiente arenga:

Estamos distribuidos en todas las tierras, bajo todos los climas. Somos hombres subterráneos, algo así como polillas del acero. Roemos el cemento de la actual sociedad. Lo roemos despacio, pacientemente. [ . . . ] Nos hemos infiltrado como lepra en todas las capas de la humanidad. Somos indestructibles. Crecemos día por día, insensiblemente. [. . .] Revestimos mil aspectos. Somos los omnipotentes $(L l$, p. 254).

En medio de la exaltación que les producen sus propias fantasías todopoderosas, Erdosain, el Astrólogo, el Buscador de Oro, olvidan el propósito inicial de su empresa e inventan ser una suerte de élite que maneja, para el "rebaño", "la divina bazofia" ( $S l$, p. 94), y que, para mantener su dominio, pretende llevar a cabo una feroz explotación, peor que la que dicen combatir. En efecto, al dinero, producto de los prostíbulos y primer financiamiento de la futura revolución, el Astrólogo agrega otras fuerzas de explotación: "Explotaremos la usura. . . la mujer, el niño, el obrero, los campos y los locos [ . . .]. Llevaremos engañados a los obreros, y a los que no quieran trabajar en las minas los mataremos a latigazos" ( $S l$, p. 96). Estas fantasías en las que triunfan sin dificultad los "locos" de las novelas constituyen un triunfo efímero que no logra ocultar la desoladora realidad de sus existencias fracasadas, marginadas, y de su radical impotencia a la hora de actuar ${ }^{11}$. $\mathrm{Si}$ se adhieren irracional y compulsivamente a la Sociedad Secreta y al plan del Astrólogo, sin comprometerse en el fondo con la empresa trascendental que se supone es una revolución, es por la naturaleza misma de dicho proyecto. Entre tantas invenciones, mentiras, farsas, la propuesta revolucionaria del Astrólogo se asemeja en conjunto a una simulación más, de una eficacia no obstante de distinta magnitud: "Yo sé que no puede ser, pero hay que proceder como si fuera factible"' ( $\mathrm{Sl}$, p. 94; nosotros subrayamos). Esta réplica circunstancial del Astrólogo a la objeción de alguno de sus adeptos encierra en clave el carácter de las soluciones planeadas por la Sociedad Secreta y su cabecilla. No se trata por lo tanto de hacer la revolución, sino simplemente, como lo admite el mis-

${ }^{11}$ Los inventos del protagonista (en particular, la "rosa de cobre") pertenecen al mismo ámbito imaginario que el proyecto de la Sociedad Secreta: nunca llevados totalmente a la práctica, existe el mismo afán por singularizarse, por ser reconocido por medio de los mismos. 
mo Astrólogo, de sustituir la acción de la cual son sin duda todos incapaces, por un plan o un proyecto que vuelve "factible" en la fantasía, y de un modo mágico, la tan anunciada revolución, que le da en suma "a lo falso la consistencia de lo cierto" ( $S l$, p. 114), gracias al indiscutible carisma verbal de su jefe. Proyecto que difícilmente, sin embargo, puede salvar la distancia que media entre la palabra o el discurso y la acción. Con un sentido próximo a la réplica anterior del Astrólogo, el protagonista, Erdosain, observa, en uno de sus monólogos, que "el poder hacerlo [se refiere a uno de sus delirios de destrucción total de la humanidad] le quitaría interés al asunto" ( $S l$, p. 54). Esta reflexión no deja lugar a dudas: se escoge lo imaginario por el tipo de respuesta (mejor tal vez sería decir por la ausencia de respuesta) que éste supone. En este contexto, el asesinato simulado de Barsut - una suerte de doble del protagonista - decidido a última hora por el Astrólogo, resulta emblemático del proyecto de revolución en su totalidad: el simulacro de muerte ocupa el lugar de la acción verdadera. Resulta irónico por cierto que el Astrólogo, que sueña con ser un "hombre de acción" (Sl, p. 160), capaz de planear innumerables formas de muertes violentas, se revele indeciso a la hora de llevar a cabo la de un solo hombre. En cuanto surgen los primeros tropiezos, el grupo se dispersa antes siquiera de dar álgunos de los pasos que conduzcan a la realización del proyecto. Planean, pues, una revolución imposible en el orden de los hechos, pero viable en el orden ilimitado de lo imaginario. Frente a la ciudad "desconocida" ( $L l$, p. 290) de la que habla Erdosain, frente al "terror" ( $S l$, pp. 7-9) que recorre sus calles y al no ser en que los confina la sociedad, en Temperley, en la casa del Astrólogo, y concretamente en el espacio que abre en la novela el asunto de la Sociedad Secreta, el protagonista y los demás integrantes del grupo dejan de ser los personajes anónimos y acosados que deambulan por la ciudad para convertirse, por arte de magia, en atractivos y poderosos "jefes": Erdosain, en "Jefe de Industrias", el Buscador de Oro, en "Jefe de Colonias y Minas", el Rufián, en "Jefe de los Prostíbulos" o en el "Gran Patriarca Prostibulario". Esta nueva identidad, fingida y fantasiosa como la revolución que pretenden llevar a cabo, es subrayada por el uso de los seudónimos que, en algunos casos, como en el del Buscador de Oro, toma el lugar del nombre que nunca nos es revelado. Sólo en las últimas páginas de Los lanzallamas, nos enteramos, mediante un recorte de periódico, que el famoso y prestigioso Astrólogo se llama, simplemente, Alberto Lezin, nombre que lo resti- 
tuye en forma abrupta a una cotidianidad vulgar, sin relieve. Los papeles representados, los títulos y los seudónimos, otorgan a los "locos" un disfraz, una identidad prestada, preferible sin embargo a no ser nadie. Leída desde este ángulo, la simulación, más allá del juego intrascendente que aparenta ser en una primera aproximación, resulta sinónima de identidad: simulan "ser" para existir mínimamente ${ }^{12}$. Lejos de constituir una alternativa viable que intente en verdad actuar y transformar la realidad hostil y enajenante de los personajes, la eficacia de esa revolución parece ser, fundamentalmente, de orden simbólico: los personajes viven de un modo imaginario las fantasías que giran en torno a una sociedad futura, seducidos por la magia que emana de las palabras del Astrólogo, quien, con su "magnífica locura", evoca ante ellos una “tierra de posible renovación" ( $S l$, pp. 96-97) ${ }^{13}$. Mucho más parecido en definitiva a un mago o a un hechicero que al político o revolucionario en que se ha querido encerrarlo ${ }^{14}$, el Astrólogo, al igual que aquél, se transforma, en el seno del grupo, en un "con-

${ }^{12}$ La simulación es recurrente en toda la obra de Roberto Arlt y merecería desde luego una exploración más detenida, que los objetivos del presente trabajo no nos permiten llevar a cabo. Digamos, pese a todo, que el acercamiento que proponemos entre simulación e identidad se ve confirmado en algunos de sus cuentos, como por ejemplo, "El traje del fantasma" (incluido en el libro de cuentos El jorobadito, Bruguera, Barcelona, 1981) o en sus piezas teatrales, Saverio el cruel y El desierto entra a la ciudad. En estos casos extremos, se combina peligrosamente la simulación o la representación de papeles falsos con la locura.

${ }^{13}$ Coincidimos en buena medida con la lectura que lleva a cabo BÁRBARA KoczaUer sobre los "locos" de la novela, a los que define como "los outsiders de una sociedad fundamentalmente pequeño burguesa" ("La rebelión de los intelectuales en Los siete locos y Los lanzallamas. Sobre la relación entre literatura y sociedad en Roberto Arlt'", en José Morales Saravia (ed.), Homenaje a Alejandro Losada, Latinoamericana, Berlín, 1986, pp. 17-36). Destaca también el "fantástico mundo alternativo" con el que los "locos" intentan resolver su crisis de identidad social. Con ello se separa de buena parte de la crítica arltiana - por ejemplo el estudio ya clásico de Diana Guerrero, Roberto Arlt, el habitante solitario, Granica Editor, Buenos Aires, 1972- que suele ver en los personajes más representativos de Arlt una imagen "del pequeño burgués deformado o a la pequeña burguesía amenazada por el descenso en el lumpen proletariado". Más discutibles nos parecen, en cambio, las similitudes que establece Koczauer entre el grupo de los "locos" y el exclusivo círculo de la vanguardia artística argentina de los años veinte.

${ }^{14}$ No se trata sin embargo de una simple imagen. Entre las habilidades o técnicas del shamán destaca, precisamente, la de ser un simulador experimentado. Cf. LÉVI-STRAuSs, "Le sorcier et sa magie", en Anthropologie structurale, Plon, Paris, 1958, p. 192. 
ductor iluminado de la angustia común"'15. Como lo hemos visto, no se dirige al raciocinio de sus seguidores, sino a su capacidad imaginativa con procedimientos seductores, cercanos a la magia: conjuros, falsos prodigios, etc., procedimientos olvidados por la modernidad técnica, mecánica. No es por lo tanto extraño que en la ciudad del futuro soñada por uno de los "locos", "toda ciencia será magia" ( $S l$, p. 180). Entre las historias individuales de los "locos", centradas en torno a una identidad problemática, y la utopía o el sueño de liberación que proyecta en el espacio narrativo el asunto de la Sociedad Secreta, no hay contradicción. La solución total que ofrece el Astrólogo sirve de contrapunto imaginario a sus existencias vacías, malogradas, y a sus deseos frustrados de ser.

Por último, diremos que frente a una crítica empeñada en desentrañar las "ideas" del autor, hemos creído importante subrayar, a lo largo de nuestro análisis, que las respuestas de Arlt, como ocurre en cualquier auténtico creador, no se dejan reducir fácilmente a esquemas o tesis. Lo que interesa en definitiva no es la verosimilitud o no del plan revolucionario y de la Sociedad Secreta sino - parafraseando al Astrólogo- la verdad de la mentira o sea la verdad de la ficción que sólo puede ser una verdad poética, imaginativa. Al poner de manifiesto sus carencias, en particular su desorientación y precaria identidad, la aventura colectiva de los "locos" anuncia al hombre anónimo de la modernidad y a la vez prefigura la crisis del mundo actual, mundo caótico, sin valores, que Arlt descifra más allá de sus signos aparentes.

Rose Corral

El Colegio de México

15 Lo dice GÉZa RÓHEIM en The origin and function of culture, Nueva York, 1943, p. 51; citado por J. Campbell, El héroe de las mil caras. Psicoanálisis del mito, F.C.E., México, 1972, p. 97. 